\title{
Hábitos e práticas alimentares de hipertensos e diabéticos: repensando o cuidado a partir da atenção primária ${ }^{1}$
}

\author{
Dietary habits of hypertensive and diabetic \\ patients: rethinking patient care \\ through primary care
}

Rosângela Minardi Mitre COTTA

Roberta Sena REIS²

Kelly Cristina Siqueira BATISTA2

Glauce DIAS²

Rita de Cássia Gonçalves ALFENAS²

Fátima Aparecida Ferreira de CASTRO $^{2}$

\section{R E S U M O}

\section{Objetivo}

Identificar hábitos e práticas alimentares inadequados apresentados por hipertensos e diabéticos usuários da Estratégia Saúde da Família, de Teixeiras (MG), visando ao desenvolvimento de estratégias de intervenção em saúde.

\section{Métodos}

Estudo descritivo de delineamento transversal, utiliza questionários semi-estruturados para entrevistar 10,3\% dos hipertensos e 15,0\% dos diabéticos, selecionados de forma probabilística e aleatória.

\section{Resultados}

Houve uma predominância de mulheres (74,4\%), de idosos (média=63,59, DP=13,12 anos) e de pessoas com baixa renda (mediana=0,5 salário-mínimo). Os hipertensos e os diabéticos apresentaram, res-pectivamente, pequeno fracionamento das refeições (mediana=3,0/4,0), elevado consumo per capita diário de açúcar $(M=165,63 \mathrm{~g}, D P=118,93$ e 105,13g, $D P=48,7)$, sal $(M=22,63 \mathrm{~g}, D P=22,26$ e $M=12,96 \mathrm{~g}, D P=16,73)$, óleo $(\mathrm{M}=64,13 \mathrm{~mL}, \mathrm{DP}=38,09$ e $\mathrm{M}=61,29 \mathrm{~mL}, \mathrm{DP}=35,57)$ e banha de porco (18,8\% e 13,3\%). Entretanto, 97,3\% e $96,7 \%$ não adicionavam sal às preparações prontas, $72,5 \%$ e $86 \%$ utilizavam somente óleo vegetal para preparar as refeições e 15,4\% e 90,0\% utilizavam adoçante artificial.

1 Apoio: Ministério da Saúde (Convênio no 517544 - PORT. 424/2004) e Fundação de Amparo à Pesquisa do Estado de Minas Gerais (Processo no 075/05).

2 Universidade Federal de Viçosa, Departamento de Nutrição e Saúde. Av. P.H. Rolfs, s/n., Campus Universitário, 36570-000,

Viçosa, MG, Brasil. Correspondência para/Correspondence to: R.M.M. COTTA. E-mail: <rmmitre@ufv.br>. 
824 | R.M.M. cOTTA et al.

\section{Conclusão}

Estratégias de cuidado em saúde devem ser desenvolvidas para incentivar mudanças nestes hábitos, objetivando a prevenção e o controle das complicações relacionadas a estas morbidades.

Termos de indexação: Atenção primária à saúde. Diabetes mellitus. Hábitos alimentares. Hipertensão. Saúde da família.

\section{A B S T R A C T}

\section{Objective}

This study aims to identify inappropriate dietary habits shown by hypertensive and diabetic patients registered in the Family Health Strategies Program, in the city of Teixeiras (MG), Brazil, aiming towards the development of health intervention strategies.

\section{Methods}

In this descriptive, cross-sectional study, semi-structured questionnaires were used to interview $10.3 \%$ of the hypertensive and $15.0 \%$ of the diabetic patients, which were probabilistically and randomly selected.

\section{Results}

Most (74.4\%) of these patients were older women (mean age of 63.59, SD=13.12 years) with low income (an average of 0.5 minimum wage). These hypertensive and diabetic patients had a small number of daily meals (mean=3.0/4.0) and consumed high amounts of sugar $(M=165.63 \mathrm{~g}, S D=118.93$ and $M=105.13 \mathrm{~g}, S D=48.7)$, salt $(M=22.63 \mathrm{~g}, S D=22.26$ and $M=12.96 \mathrm{~g}, S D=16.73)$, oil $(M=60 \mathrm{~g}, S D=43.23)$, and lard (18.8\% and 13.3\%) daily. However, $97.3 \%$ and $96.7 \%$ respectively did not add salt to the ready-to-eat preparations, $72.5 \%$ and $86 \%$ respectively used only vegetable oil to prepare their meals, and $15.4 \%$ and $90 \%$ respectively of the interviewed patients used artificial sweetener.

\section{Conclusion}

Heath care strategies need to be developed to stimulate changes in these habits to prevent and control the complications related to these morbidities.

Indexing terms: Primary health care. Diabetes mellitus. Dietary habits. Hypertension. Family health.

\section{N T R O D U ÇÃ O}

O crescimento epidêmico das Doenças Crônicas Não Transmissíveis (DCNT), principalmente doenças cardiovasculares e Diabetes Mellitus (DM) do tipo 2, pode ser atribuído ao aumento da exposição aos principais fatores de risco para essas doenças e às mudanças na pirâmide demográfica, com o aumento da longevidade da população. Além disso, a maioria dos países em desenvolvimento passa por uma transição nutricional, acarretando o aumento expressivo da obesidade, o que também explica o crescimento da incidência das DCNT¹.

Em 2003, estimava-se que havia, aproximadamente, 150 milhões de diabéticos no mundo, sendo previsto o dobro do número de portadores desta doença no ano de $2025^{2}$. De acordo com o Estudo Multicêntrico, realizado em nove capitais brasileiras sobre a prevalência do $\mathrm{DM}^{3}$, existem no País 7,6\% de indivíduos diabéticos na faixa etária de 30 a 69 anos de idade. No Brasil, a mortalidade por doenças crônicas aumentou significantemente, sendo considerada a principal causa de morte em vários estados. Neste contexto, o diabetes encontra-se entre as 10 causas principais de óbitos no País ${ }^{4}$. Estima-se que 11,0\% da população brasileira com mais de 40 anos seja portadora de DM. Tal coeficiente de prevalência traduz a gravidade do problema e fornece subsídios para o planejamento das ações de saúde que visem à prevenção ou ao controle da doença ${ }^{5}$.

O Ministério da Saúde desenvolveu uma campanha por meio do Plano Nacional de Reorganização da Atenção à Hipertensão Arterial e ao Diabetes mellitus (PRAHD) objetivando, em sua 
primeira fase (realizada no período de março a abril de 2001), promover um rastreamento na comunidade com pessoas apresentando idade de 40 anos ou mais e com usuários do Sistema Único de Saúde (SUS), para a detecção daqueles indivíduos suspeitos de portarem DM e Hipertensão Arterial $(\mathrm{HA})^{6}$.

Ao analisarem os dados do rastreamento nacional (valores glicêmicos e itens relacionados à saúde, obtidos por meio de inquéritos), Georg et al. 7 identificaram um número provável de novos casos de DM equivalente a 1350255 . Pressupondo que um terço dos participantes que apresentaram testes positivos fizeram a confirmação do diagnóstico posteriormente, esse número passaria para, aproximadamente, 518579 indivíduos.

Em Minas Gerais, foram realizados 2874377 exames, representando uma cobertura de $84,5 \%$. Desses, 418621 apresentaram resposta glicêmica alterada, valor este que corresponde a três vezes o número de portadores que eram cadastrados no Programa de Diabetes até o ano de 2001, em todo o Estado. Esses resultados demonstram que a maior parte dos diabéticos encontrava-se sem diagnóstico, o que é esperado pela história natural da doença, que pode ser oligossintomática por vários anos.

Ao receberem o diagnóstico de DM do tipo 2, muitos indivíduos já apresentam alguma complicação crônica da doença, que, além de onerar o sistema de saúde, compromete a qualidade de vida destes indivíduos. Assim, o diagnóstico precoce da doença é fundamental para que o controle glicêmico seja atingido e a manifestação de complicações, como a hipertensão arterial e dislipidemia, seja prevenida ${ }^{9}$.

A HA apresenta-se como um dos problemas de saúde de maior prevalência na atualidade ${ }^{10}$, representando o tipo de morbidade mais freqüente na população brasileira ${ }^{11}$. Definida pela Organização Mundial de Saúde (OMS) como elevação crônica da pressão arterial sistólica e/ou pressão arterial diastólica ${ }^{12}$, a HA vem atingindo cada vez mais pessoas adultas, em especial os mais idosos, e adultos jovens, estimando-se que atinja, aproximadamente, $22 \%$ da população brasileira acima de $20 \operatorname{anos}^{10}$. A prevalência de hipertensão na população urbana adulta brasileira varia, de acordo com as $\vee$ Diretrizes Brasileiras de Hipertensão Arterial, de 22,3\% a 43,9\%, sendo maior nas Regiões Nordeste (7,2\% a 40,3\%) e Sudeste $(5,5 \% \text { a } 32,7 \%)^{13}$. Essas prevalências são similares às de países desenvolvidos, como os Estados Unidos, onde, entre os anos de 1999 e 2000, 28,7\% da população adulta era hipertensa ${ }^{14}$.

Segundo Luz \& Cesena ${ }^{15}$, o tratamento de Doenças Cardiovasculares (DCV) não é encarado com a seriedade devida; pela prevenção, pode-se reduzir o risco modificável desta e de outras doenças como a hipertensão arterial e diabetes, além de favorecer para a diminuição do custo social das mesmas. No Brasil, as DCNT foram responsáveis pela maior parcela dos óbitos e das despesas com assistência hospitalar no Sistema Único de Saúde, totalizando cerca de $69 \%$ dos gastos com atenção à saúde em 2002. Desde a década de 60, as doenças cardiovasculares vêm liderando as causas de óbitos no Brasil. Atualmente, elas são a causa básica de morte de cerca de dois terços do total de óbitos por causas conhecidas no Brasil 1,16,17.

É importante destacar que as DCNT são de etiologia multifatorial e compartilham de vários fatores de riscos modificáveis, como o tabagismo, a obesidade, a dislipidemia, a inatividade física e a alimentação inadequada. Estudos epidemiológicos ressaltam que as DCV seriam, por exemplo, uma causa relativamente rara de morte na ausência destes principais fatores de risco ${ }^{1,17}$.

De acordo com Stamber et al. ${ }^{18}$, cerca de $75 \%$ dos casos novos de doenças ocorridos em países desenvolvidos nas décadas de 70 e 80, poderiam ser explicados pela ingestão de dieta inadequada e pela inatividade física.

Destaca-se que a ingestão de uma alimentação adequada é importante para que o controle do diabetes e da hipertensão seja atingido. São necessárias mudanças dos hábitos alimentares, 
favorecendo um melhor controle metabólico, do peso corporal e da pressão arterial ${ }^{19}$. No entanto, como esses hábitos têm geralmente suas bases fixadas dentro do núcleo familiar, ainda na infância, os mesmos são difíceis de serem modificados, o que torna a intervenção do nutricionista importante, com vistas à conscientização e ao empoderamento da população, considerando os aspectos psicológicos, socioculturais, educacionais e econômicos $^{20}$.

A transição epidemiológica, demográfica e nutricional no País deve servir de incentivo para o desenvolvimento de estratégias de promoção da saúde, de prevenção e controle dos principais fatores de risco comuns ${ }^{1,17}$. Partindo do pressuposto que as medidas de educação em saúde não devem ser autoritárias e normativas, mas sim dialógicas ${ }^{21}$, destaca-se a Estratégia Saúde da Família (ESF), como locus para trabalhar estas questões junto à comunidade. Na ESF, os profissionais de saúde devem estar capacitados para prestar assistência integral à população. O cuidado em saúde deve ser participativo e humanizado, visto que é imprescindível integrar o saber técnico ao saber popular para melhor direcionar as ações de saúde, intervir de forma mais efetiva, ao envolver a população e/ou os grupos populacionais no processo de cuidado, proporcionar mudança no estilo de vida e melhoria na qualidade de vida dos indivíduos e famílias. O conhecimento técnico-científico nem sempre deverá ser transmitido a todos que dele necessitam da mesma forma; é importante adaptá-lo à realidade de cada indivíduo e dos grupos populacionais de maneira que este possa ser aplicado em conformidade à realidade socioeconômica e educacional e, dessa forma, associado a algum aspecto da vida e da família desses indivíduos ${ }^{21-23}$.

As estratégias que envolvem a alimentação e nutrição, como formas de intervenção, tornam-se imprescindíveis a qualquer programa que vise, a partir do princípio da integralidade das ações, elevar a qualidade de vida da população ${ }^{24}$. A efetividade de políticas de promoção de vida saudável requer a participação dos diversos setores e atores sociais responsáveis e comprometidos com a saúde e qualidade de vida da população brasileira'.

Diante disso, este trabalho teve como objetivo avaliar os principais aspectos relacionados aos hábitos e às práticas alimentares de usuários diabéticos e hipertensos cadastrados na Equipe de Saúde da Família, de Teixeiras (MG), visando à identificação de inadequações alimentares e de estilo de vida para o desenvolvimento de estratégias de promoção da saúde, prevenção e controle dos principais fatores de risco comuns a estas morbidades.

\section{MÉ TODOS}

O município de Teixeiras se localiza na região da Zona da Mata mineira e, segundo estimativas do Instituto Brasileiro de Geografia e Estatística (IBGE), a população total deste município, estimada em 2005, era de 11854 habitantes. Dados do Sistema de Informação da Atenção Básica (SIAB) demonstram que em abril de 2005, foram cadastrados 1451 hipertensos e 200 diabéticos na ESF deste município ${ }^{25}$.

Trata-se de um estudo descritivo de delineamento transversal, no qual foram entrevistadas 180 pessoas, representando $10,3 \%$ da população de Teixeiras com hipertensão e 15,0\% da população com diabetes. Participaram do presente estudo 150 usuários diagnosticados como hipertensos e 30 como diabéticos.

A amostra, representativa de hipertensos e diabéticos cadastrados na ESF de Teixeiras (MG), foi selecionada segundo a base de amostragem dos cadastros dos prontuários médicos da ESF, de forma probabilística e aleatória, seguindo os critérios recomendados por Hulley et al. ${ }^{26} \mathrm{e}$ Richardson ${ }^{27}$, uma vez que, a seleção guiada pelas leis da probabilidade e por fórmulas apropriadas para o cálculo do tamanho da amostra, estabelece uma chance adequada de ser representativa da população. Inicialmente previu-se um calculo de 15,0\% tanto para hipertensos como para diabé- 
ticos. No caso dos hipertensos, houve uma perda de $4,6 \%$, devido a recusas dos usuários em participar do estudo.

O instrumento de coleta de dados utilizado nesta pesquisa foi um questionário semi-estruturado elaborado pela equipe de pesquisadores, autores deste estudo, a partir de trabalhos presentes na literatura científica. Dentre as variáveis analisadas, destaca-se: idade, sexo, renda, aspectos relacionados aos hábitos e práticas alimentares desta população, tais como: número de refeições diárias, uso de adoçantes artificiais, adição de sal a preparações ou alimentos já prontos, tipo de gordura utilizada nas preparações, quantidade de água ingerida diariamente, orientações sobre cuidados com a alimentação, consumo per capita diário de açúcar, sal e óleo, dentre outros aspectos.

Vale ressaltar que estes valores per capita foram relatados pelos entrevistados a partir da disponibilidade da lista de compras mensal, o que, indiretamente, reflete o consumo per capita destes alimentos. Mais especificamente, foi perguntado a respeito da quantidade mensal adquirida e consumida destes alimentos pela família. Por exemplo, número de latas de óleos, quilos de açúcar e sal em relação ao número de pessoas residentes no domicílio que consomem estes alimentos, permitindo, assim, estimar as quantidades per capita diárias consumidas destes alimentos. A quantidade de água ingerida diariamente foi obtida a partir de relatos dos usuários, e para confirmar estes valores a ingestão, também, era questionada em medida caseira para, posteriormente ser transformada em $\mathrm{mL}$.

No presente estudo, utilizou-se a Análise da Estratégia Global Sobre Alimentação Saudável, Atividade Física e Saúde da Organização Mundial de Saúde, a Pirâmide Alimentar Adaptada: Guia para Escolha dos Alimentos, o Plano de Reorganização da Atenção à Hipertensão Arterial e ao Diabetes mellitus e o Guia Alimentar do Ministério da Saúde (MS) como referenciais teóricos para avaliar os principais aspectos relacionados aos hábitos e às práticas alimentares da população estudada ${ }^{1,19,28,29}$.
Previamente, realizou-se um estudo piloto em população com características semelhantes às de Teixeiras, porém, em município distinto do estudado (Viçosa, MG), com o objetivo de revisar o questionário, direcionar aspectos da investigação e treinar a equipe para a aplicação do instrumento proposto 27,30 .

A coleta de dados foi realizada nas residências dos usuários por meio de entrevistas por uma equipe de pesquisadores devidamente treinados, apresentados pelos Agentes Comunitários de Saúde (ACS) durante suas visitas domiciliares. O período de coleta dos dados foi de maio a setembro de 2005. Os dados foram digitados e analisados no software Epiinfo 6.0, com auxílio do Microsoft Excel 2000, sendo realizados testes estatísticos descritivos (média, freqüência, percentuais).

Os usuários que concordaram em participar desta pesquisa assinaram um Termo de Consentimento Livre e Esclarecido. O presente projeto foi aprovado pelo Comitê de Ética em Pesquisas com Seres Humanos da Universidade Federal de Viçosa (MG), Brasil (Parecer $n^{\circ}$ 40511353607 de 16/11/2005).

A justificativa para a escolha deste município para a realização deste estudo foi em função da ampla cobertura da ESF (100\%), além do apoio recebido pela Prefeitura e pelas equipes para a realização da pesquisa.

\section{RESULTADOS}

Os participantes deste estudo eram, em sua maioria, do sexo feminino $(74,4 \%)$, a idade dos entrevistados estudados oscilou entre 14 e 93 anos, com uma média de 63,59 (Desvio-Padrão $\mathrm{DP}=13,12$ ) anos e uma mediana de 63,5 anos. $\mathrm{A}$ renda per capita mediana era de 0,5 salários-mínimos; variando de 0,08 a 2,43 salários-mínimos.

Em relação ao diagnóstico apresentado, 150 participantes eram hipertensos e 30 diabéticos $(D M 1=5$ e $D M 2=25)$. Ao estratificar os parti- 
cipantes por faixas etárias e sexo foram observados entre as faixas de 14 a 19 anos uma mulher; de 20 a 59 anos 57 mulheres e 10 homens e com idade maior ou igual a 60 anos 75 mulheres e 37 homens.

No campo das práticas alimentares, verificou-se que $60,1 \%$ dos hipertensos e $53,3 \%$ dos diabéticos tinham horta em casa.

Ao serem questionados sobre o número de refeições realizadas diariamente, obteve-se mediana igual a 3,0 (variando de um a seis) para os hipertensos e mediana igual a 4,0 (variando de um a sete) para os diabéticos.

Dentre os entrevistados, 66,9\% dos hipertensos e 100,0\% dos diabéticos informaram evitar algum tipo de alimento. Dentre os alimentos mais citados pelos hipertensos e diabéticos, respectivamente, destacam-se, principalmente: gordurosos (44,4\% e 20,0\%), alimentos ricos em carboidratos, como massas, bolo, arroz, angu (17,7\% e $73,3 \%)$, carne suína $(24,2 \%$ e $10,0 \%)$ e sal $(20,2 \%$ e 6,6\%), como pode ser visto na Tabela 1. Vale ressaltar que o entrevistado poderia atribuir mais de uma resposta à pergunta dirigida.

As principais causas relatadas pelos hipertensos e diabéticos entrevistados, respectiva- mente, para que tais alimentos fossem evitados foram: a prevenção ou o tratamento das doenças apresentadas, principalmente, diabetes ou medo de se tornar diabético $(5,0 \%$ e 46,6\%), dislipidemias (21,2\% e 13,3\%), hipertensão arterial ou medo de se tornar (15,1\% e 6,6\%). Não obstante, 21,2\% dos hipertensos e 13,3\% dos diabéticos sabiam que os alimentos citados podiam causar algum mal à saúde, mas não souberam relacioná-lo a nenhuma doença. As demais causas encontram-se detalhadas na Tabela 2.

O consumo per capita diário médio de açúcar, sal e óleo dos hipertensos entrevistados correspondeu a 165,63g ( $\mathrm{DP}=118,93) ; 22,63 \mathrm{~g}$ $(D P=22,26)$ e $64,13 \mathrm{~mL}(D P=38,09)$. Dentre os diabéticos o consumo per capita correspondeu a $105,13 \mathrm{~g}(\mathrm{DP}=48,7) ; 12,96 \mathrm{~g}(\mathrm{DP}=16,73)$ e $61,29 \mathrm{~mL}$ $(\mathrm{DP}=35,57)$ respectivos a açúcar, sal e óleo.

Além disso, verificou-se que a substituição do açúcar pelo adoçante artificial era feita por 15,4\% dos hipertensos e por 90,0\% dos diabéticos, com predominância do adoçante à base de sacarina sódica e ciclamato de sódio (100,0\%).

Em relação ao tipo de gordura utilizada para o preparo das refeições, houve predomínio no uso de óleo vegetal, sendo este de $72,5 \%$ e

Tabela 1. Alimentos evitados pelos hipertensos e diabéticos usuários da estratégia saúde da família município de Teixeiras (MG), Brasil, 2005.

\begin{tabular}{lcc}
\hline Alimentos evitados & Hipertensos (\%) & Diabéticos (\%) \\
\hline Gordura & 44,44 & 20,00 \\
Carboidratos (massas, bolo, arroz, angu) & 17,70 & 73,33 \\
Carne suína & 24,24 & 10,00 \\
Doces & 10,10 & 23,33 \\
Sal & 20,20 & 6,66 \\
Laticínios & 6,06 & 3,33 \\
Ovos & 6,06 & 3,33 \\
Verduras e legumes & 14,14 & 6,66 \\
Carne vermelha & 7,07 & 0,00 \\
Enlatados/conservas & 5,05 & 0,00 \\
Pimenta & 5,05 & 0,00 \\
Frutas & 4,04 & 0,00 \\
Manteiga & 2,02 & 0,00 \\
Café & 1,01 & 0,00 \\
Refrigerantes & 1,01 & 0,00 \\
\hline Total & 168,19 & 146,64 \\
\hline
\end{tabular}


Tabela 2. Principais causas para que os hipertensos e os diabéticos usuários da estratégia saúde da família evitassem algum tipo e alimento. Município de Teixeiras (MG), 2005

\begin{tabular}{|c|c|c|}
\hline Motivos para não seguir as orientações & Hipertensos (\%) & Diabéticos (\%) \\
\hline Diabetes ou medo de se tornar diabético & 5,05 & 46,66 \\
\hline Fazer mal à saúde & 21,21 & 13,33 \\
\hline Dislipidemias & 21,21 & 6,66 \\
\hline Hipertensão arterial ou medo de se tornar & 15,15 & 6,66 \\
\hline Prescrição médica & 11,11 & 3,33 \\
\hline Não gostar & 8,08 & 10,00 \\
\hline Afetar o fígado & 4,04 & 0,00 \\
\hline Engorda & 3,03 & 3,33 \\
\hline Fator econômico & 0,00 & 3,33 \\
\hline Perda de peso & 0,00 & 3,33 \\
\hline Enjôo & 0,00 & 3,33 \\
\hline Gastrite e úlcera & 4,04 & 0,00 \\
\hline Estômago & 2,02 & 0,00 \\
\hline Queimação & 1,01 & 0,00 \\
\hline Intestino & 3,03 & 3,03 \\
\hline Estase gástrica & 3,03 & 0,00 \\
\hline Alergia & 2,02 & 0,00 \\
\hline Entope veias & 2,02 & 0,00 \\
\hline Hemorróidas & 1,01 & 0,00 \\
\hline Gota & 1,01 & 0,00 \\
\hline Problema renal & 1,01 & 0,00 \\
\hline Dor de cabeça & 1,01 & 0,00 \\
\hline
\end{tabular}

$86 \%$ entre os hipertensos e os diabéticos, respectivamente. No entanto, entre os entrevistados houve uma proporção de 18,8\% e 13,3\% quanto ao consumo de óleo vegetal juntamente à banha de porco, e à utilização somente de banha de porco por $8,7 \%$ dos hipertensos entrevistados.

Um ponto positivo é que $97,3 \%$ dos hipertensos e $96,7 \%$ dos diabéticos informaram que não adicionavam sal às preparações ou aos alimentos depois de prontos.

A mediana da ingestão de água foi igual a $480 \mathrm{~mL}$ e $550 \mathrm{~mL}$ ao dia entre os hipertensos e os diabéticos, respectivamente, variando entre 0 e $3 \mathrm{mil} / \mathrm{mL}$. Muitos dos participantes deste estudo informaram ingerir água apenas para auxiliar na administração dos medicamentos.

Mais da metade dos entrevistados, 51,0\% dos hipertensos e $80,0 \%$ dos diabéticos, relataram terem sido orientados quanto à importância da ingestão de uma alimentação saudável, receben- do tais informações principalmente de médicos (77,6\% dos hipertensos e $75,0 \%$ dos diabéticos), como demonstrado na Figura 1. Vale ressaltar que o entrevistado poderia atribuir mais de uma resposta à pergunta dirigida.

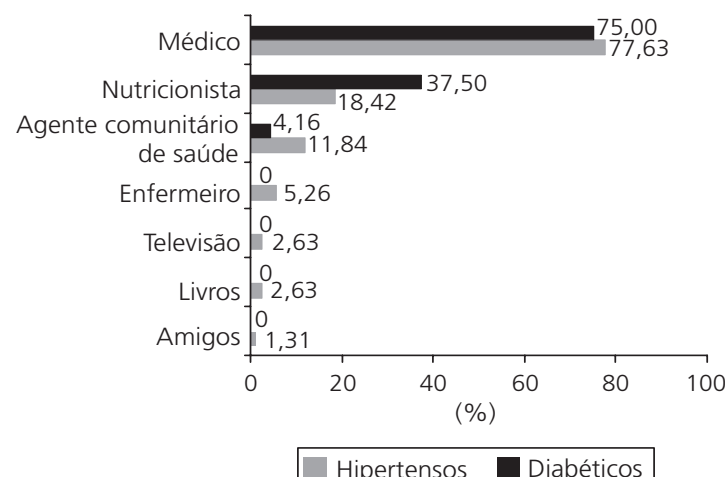

Figura 1. Fontes de informações obtidas sobre alimentação saudável relatadas pelos usuários entrevistados da estratégia saúde da família município de Teixeiras (MG), 2005. 
830 | R.M.M. COTTA et al.

Tabela 3. Motivos dos hipertensos e diabéticos usuários da estratégia saúde da família não seguir as orientações. Município de Teixeiras (MG), 2005.

\begin{tabular}{lcc}
\hline Motivos para não seguir as orientações & Hipertensos (\%) & Diabéticos (\%) \\
\hline Não soube responder & 29,42 & 40,00 \\
Hábitos & 23,52 & 10,00 \\
Não acredita & 17,64 & 10,00 \\
Fatores econômicos & 17,64 & 30,00 \\
Falta de acesso aos alimentos & 5,89 & 0,00 \\
Força de vontade & 5,89 & 0,01 \\
Falta de apetite & 0,00 & 0,00 \\
\hline Total & 100,00 & 100,00 \\
\hline
\end{tabular}

Embora tenham recebido as orientações, $22,4 \%$ dos hipertensos e $40,0 \%$ dos diabéticos afirmaram não seguir tais orientações, sendo os principais motivos atribuídos a isto os hábitos ( $23,5 \%$ dos hipertensos e $10,0 \%$ dos diabéticos), fatores econômicos ( $17,6 \%$ dos hipertensos e $30 \%$ dos diabéticos) e não acreditar ( $17,7 \%$ dos hipertensos e $10,0 \%$ dos diabéticos), como detalha a Tabela 3.

\section{I S C U S S Ã O}

Um dado relevante encontrado neste estudo foi o baixo número de refeições diárias (mediana $=3$ e mediana $=4$ ) realizadas pelos hipertensos e diabéticos, respectivamente. O maior fracionamento de refeições constitui importante medida de prevenção e controle da dislipidemia e, conseqüentemente, de prevenção para doenças cardiovasculares. Ensaio clínico realizado com mulheres hiper-colesterolêmicas, com excesso de peso (índice de massa corporal $>25 \mathrm{~kg} / \mathrm{m}^{2}$ ), não fumantes, com idade entre 30 e 50 anos, demonstrou o efeito do aumento do número de refeições, em indivíduos que realizavam três ou menos refeições diárias, na redução do colesterol total sérico. Esta correlação foi verificada independentemente do peso, da idade e do tipo de alimento adicionado à dieta (frutas ou biscoito de

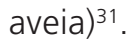

Metade dos entrevistados do presente estudo tinha horta em casa, aspecto importante a ser considerado em possíveis intervenções, já que ao se tratar de uma população de baixa renda, impossibilitada de comprar alguns alimentos, este é um aspecto que deve ser enfatizado, pois assim será possível contribuir para uma melhoria da alimentação a partir do incentivo ao cultivo de hortaliças, vegetais, frutas, ou seja, alimentos saudáveis.

Mais da metade dos entrevistados (66,9\% dos hipertensos e 100,0\% dos diabéticos) relatou evitar algum tipo de alimento, geralmente decorrente da preocupação em prevenir doenças como dislipidemias, diabetes e hipertensão. Tal preocupação também foi constatada em estudo realizado com idosos, que apresentavam bom nível socioeconômico e faziam uso de alimentos dietéticos (como adoçantes, refrigerantes e leite e derivados), ao invés da restrição de alimentos que, porventura pudessem ser prejudiciais à saúde ${ }^{32}$.

Segundo os entrevistados, os principais alimentos evitados eram os gordurosos, ricos em carboidratos, carne de porco, sal, além de doces, hortaliças, laticínios, pimenta, carne vermelha e conservas. Segundo o Ministério da Saúde ${ }^{19}$, a ingestão de alimentos ricos em gordura saturada e colesterol, como carne de porco, embutidos, miúdos, vísceras, pele de frango, dobradinha, gema de ovo, mocotó, carne vermelha com gordura aparente, leite e iogurte integral, manteiga, creme de leite, chocolate deve ser evitada. Também se recomenda evitar frituras e, para diabéticos, sobretudo, evitar o consumo excessivo de 
carboidratos, como açúcar, mel, garapa, rapadura e doces em geral. No entanto, hoje, sabe-se que o consumo dos alimentos ricos em carboidratos, como os citados anteriormente, não eleva mais a glicemia do que a ingestão de quantidades equivalentes de carboidratos complexos ${ }^{33}$. Tal afirmação é baseada no valor do índice glicêmico desses alimentos. Quanto maior o índice glicêmico de um alimento, maior será seu impacto na resposta glicêmica pós-prandial. De acordo com a Tabela Internacional de Valores de Índice Glicêmico e Carga Glicêmica, enquanto alimentos fontes de carboidrato simples, como os citados anteriormente, têm um índice glicêmico médio de 64 , o valor médio deste índice para os alimentos fonte de carboidratos complexos equivale a $62^{34}$.

A OMS recomenda um aumento do consumo de frutas, verduras, legumes, cereais integrais e nozes, por estes alimentos substituírem outros de alto valor energético e baixo valor nutricional, como cereais e grãos processados, e açúcar refinado, básicos na preparação de alimentos industrializados e fast food. Estes alimentos podem introduzir nutrientes com efeitos significativos na saúde dos indivíduos, especificamente na prevenção de DCNT como obesidade, doenças cardiovasculares, diabetes tipo ${ }^{21}$.

No que se refere ao consumo de adoçantes artificiais, foi relatado o seu uso por $15,4 \%$ dos hipertensos e por $90,0 \%$ dos diabéticos, com uma preferência absoluta $(100,0 \%)$ pelos adoçantes à base de sacarina sódica e ciclamato de sódio. A preferência por este tipo de adoçante foi encontrada em outro trabalho, realizado entre freqüentadores de clubes da melhor idade de Maceió (AL). Dentre os 59,0\% que afirmaram usar adoçantes artificiais de adição, 57,6\% utilizavam aqueles à base de sacarina e ciclamato e $42,4 \%$, à base de aspartame ${ }^{35}$. Castro \& Franco $^{36}$ também encontraram uma maior prevalência de indivíduos que utilizavam edulcorantes à base de sacarina e ciclamato $(45,2 \%)$, seguidos do aspartame $(29,3 \%)$, ao avaliarem 195 pacientes diabéticos de um ambulatório multidisciplinar. Deve-se ter em conta que o edulcorante à base de sacarina e ciclamato é encontrado a um preço mais acessível no mercado, talvez este fato possa explicar a preferência deste tipo de adoçante, pelo menos em parte ${ }^{36}$.

Segundo Bezerra et al. ${ }^{37}$, o uso de adoçantes constitui uma boa alternativa para diabéticos na substituição do açúcar em preparações. Neste estudo, 49 provadores não treinados avaliaram diferenças sensoriais em doces tipo beijinho, preparados com adição de açúcar e com adição de adoçante do tipo ciclamato de sódio e sacarina; sendo que 20 provadores (menos da metade) identificaram diferença no sabor dos doces. Segundo publicação do Ministério da Saúde ${ }^{19}$ o uso de adoçantes deve ser feito dentro dos limites seguros, sendo recomendado alternar o tipo utilizado periodicamente. Adoçantes como aspartame, ciclamato, sacarina, acessullfane $\mathrm{K}$ e sucralose constituem boas opções, por praticamente não contribuírem para o aporte energético. Porém, indivíduos hipertensos ou com problemas renais, devem evitar os adoçantes que contenham ciclamato de sódio, por contribuírem para o aumento do consumo de sódio. No inquérito realizado por Castro \& Franco ${ }^{36}$, foi verificado que $57,1 \%$ dos diabéticos avaliados gostavam de usar o adoçante, principalmente porque acostumaram a utilizá-lo (87,6\%). Esta é uma indicação de que a modificação dos hábitos de consumo do usuário pode ser atingida, se o mesmo for motivado para tal.

Além disso, foi encontrado no presente estudo que $97,3 \%$ dos hipertensos e $96,7 \%$ dos diabéticos afirmaram não adicionar sal à comida depois de pronta. Porém, ao verificar o gasto mensal das famílias com a compra de sal, chegou-se a um per capita diário de 22,63g ( $\mathrm{DP}=22,26)$ e $12,96 \mathrm{~g}(\mathrm{DP}=16,73)$ entre os hipertensos e os diabéticos, respectivamente. Segundo as Diretrizes Brasileiras de Hipertensão, a dieta habitual diária do brasileiro contém de 10 a 12g/dia de sal. Recomenda-se que este valor seja reduzido pela metade $(6 \mathrm{~g})$ incluindo o sal já presente naturalmente nos alimentos. Para tanto, recomenda-se reduzir o sal adicionado aos alimentos, evitar 
o saleiro à mesa e reduzir ou abolir os alimentos industrializados, como enlatados, conservas, frios, embutidos, sopas, temperos, molhos prontos e salgadinhos ${ }^{13}$. Certamente, o consumo de sal na população em estudo deverá ser um importante ponto de intervenção.

O consumo elevado de sal tem sido associado aos níveis pressóricos elevados, conforme demonstra estudo de base populacional realizado em Pelotas ${ }^{38}$. Em média, a cada redução de $3 \mathrm{~g}$ de sal, há um decréscimo de $5 \mathrm{mmHg}$ na pressão sistólica ${ }^{39}$. No entanto, a redução do consumo de sal não deve ser utilizada como uma prática isolada, visto que o contexto geral da alimentação tem tanta importância quanto à redução do sódio, potencializando os seus benefícios ${ }^{39}$. Assim, recomenda-se, também, um aumento na ingestão de potássio, pela ingestão de alimentos ricos neste nutriente (banana, melão, mamão, feijão, lentilha, vegetais de cor verde), já que estudos apontam que aumentando esse consumo pode-se prevenir o aumento da pressão arterial e preservar a circulação cerebral ${ }^{8}$. O sódio e o potássio são minerais essenciais para a regulação dos fluidos intra e extracelulares, atuando na manutenção da pressão sanguínea'.

Foram verificados, ainda, entre os hipertensos e os diabéticos, respectivamente, consumos per capitas diários de açúcar (165,63g, DP=118,93 e $105,13 \mathrm{~g}, \mathrm{DP}=48,7)$ e óleo $(64,13 \mathrm{~mL}, \mathrm{DP}=38,09$ e $61,29 \mathrm{~mL}, D P=35,57)$ muito acima das faixas recomendadas (açúcar: 27,5-55g; óleo: 8,11-16,22g) pela Pirâmide Alimentar Adaptada. Visto que já existem, na forma natural, de composição ou de adição em várias preparações, os óleos e as gorduras, os açúcares e os doces devem ser consumidos moderadamente ${ }^{28}$. A excessiva ingestão de açúcares e gorduras (principalmente as saturadas), junto com o aumento do consumo de produtos industrializados, o baixo consumo de frutas e hortaliças figuram entre as tendências desfavoráveis na dieta do brasileiro, contribuindo para a obesidade, o diabetes, as doenças cardiovasculares, algumas formas de câncer e outras doenças crônicas ${ }^{40,41}$. Em experimentos realizados com ratos alimentados com excesso de gordura, observou-se o desenvolvimento da obesidade, além do aumento da pressão arterial e a redução na ação da insulina sistêmica muscular e em adipócitos. Já outros trabalhos relataram que modificações na resistência à insulina independem da adiposidade, apesar de manter a sua correlação com a ingestão de gordura ou o tipo de gordura ingerida ${ }^{42}$.

A mediana da ingestão de água foi igual a $480 \mathrm{~mL}$ e $550 \mathrm{~mL}$ ao dia entre os hipertensos e os diabéticos entrevistados, o que se encontra bem abaixo da recomendação diária. Segundo o Ministério da Saúde, recomenda-se beber, pelo menos, 2 litros (6 a 8 copos) de água por dia, dando preferência ao consumo de água nos intervalos das refeições ${ }^{29}$.

A maioria dos entrevistados, $51,0 \%$ dos hipertensos e $80,0 \%$ dos diabéticos, relatou já ter sido orientada quanto à ingestão de uma alimentação adequada, principalmente por médicos (77,6\% dos hipertensos e 75,0\% dos diabéticos). Em estudo realizado em Ribeirão Preto (SP), em indivíduos portadores de diabetes tipo 2, verificou-se que as orientações sobre dieta, exercícios físicos e medicamentos pouco constavam (apenas 17,2\%) entre as recomendações recebidas pelos indivíduos da investigação. Além disso, essas orientações foram dadas em quase sua totalidade $(96,5 \%)$ pelo médico ${ }^{43}$. $\mathrm{O}$ incentivo à adoção de hábitos alimentares saudáveis é imprescindível para a prevenção de doenças como obesidade, hipertensão e diabetes ${ }^{8}$. Neste sentido, deve-se destacar a importância da atuação inter e multiprofissional, principalmente em unidades de saúde, que têm como princípio básico a atenção primária em saúde em áreas geográficas definidas, tendo como referência o cuidado em saúde não só dos indivíduos, mas, principalmente, de famílias, grupos populacionais e comunidades, como é o caso das ESF.

Embora Teixeiras seja um município de pequeno porte, do interior do Brasil, os dados aqui apresentados podem representar um retrato da realidade da maioria dos municípios brasileiros, 
segundo o IBGE, dos 5564 municípios existentes no País, 71\% são de pequeno porte. Diante disso, destaca-se a importância do papel da ESF no contexto de cuidado à saúde, em atenção primária, a partir do trabalho inter e multiprofissional com indivíduos, família e comunidades, em âmbito nacional e internacional ${ }^{19,44}$.

\section{O N CLUS Ã O}

Diante das práticas alimentares inadequadas constatadas no estudo - como o baixo fracionamento das refeições, o elevado consumo per capita diário de açúcar, sal e óleo, a baixa ingestão hídrica, entre outros resultados - verifica-se a importância do desenvolvimento de estratégias de educação e promoção de saúde, visando a incentivar mudanças nestes hábitos.

Nesse caso, a educação e a promoção de saúde não se referem à busca de subsídios para que a doença não se estabeleça, uma vez que no portador de diabetes mellitus e hipertensão arterial ela já se instalou; o enfoque, neste caso, refere-se à importância de que estes indivíduos se conscientizem que é possível levar uma vida saudável e normal, quando se é portador de uma doença crônica. Nesse sentido, a mudança nos hábitos de vida é de fundamental importância, não só para estes usuários da Equipe de Saúde da Família, como também para aqueles que estão ao seu redor, evitando, assim, que indivíduos pré-dispostos ao diabetes e à hipertensão desenvolvam também a doença.

Destarte, os resultados deste estudo remetem à seguinte problemática, que se coloca para os pesquisadores da área de saúde pública e nutrição social: como transferir o conhecimento produzido pelos pesquisadores/academia para os serviços de saúde, profissionais e usuários alvo do estudo, de modo a impactar e transformar a realidade encontrada? Só assim, é possível transformar o mundo real por meio de ações articuladas e comprometidas com a proposta de um serviço de saúde eficaz com pessoas (profissionais, usuários, alunos, professores e pesquisadores) mais qualificadas, bem informadas e motivadas.
A partir destas perspectivas uma das estratégias implementadas por esta equipe de trabalho foi a capacitação e a valorização dos profissionais da ESF, em especial os Agentes Comunitários de Saúde, por serem estes os profissionais que lidam mais diretamente e de forma mais rotineira (visitas domiciliares rotineiras) com a população.

Por fim, deve-se destacar que, tendo como base as informações dos usuários, iniciaram-se atendimentos nutricionais individualizados voltados especialmente para a orientação de hábitos alimentares mais adequados. Outrossim, formaram-se grupos de apoio, a partir dos quais foi possível o estabelecimento de confiança entre usuário e profissionais de saúde, fator favorável à adesão ao tratamento e à mudança de estilo de vida e hábitos alimentares.

\section{A GRADECIMENTO}

À Nicole Euclydes Nazar, pela realização da revisão gramatical e ortográfica.

\section{COLABORADORES}

R.M.M. COTTA foi responsável pela coordenação, pelo planejamento, pela orientação e pelo acompanhamento do trabalho de campo e trabalhou na redação e na revisão do manuscrito. R.S.REIS e K.C.S. BATISTA participaram do planejamento e da execução das atividades de campo e auxiliaram na redação do ma-nuscrito. G. DIAS participou do planejamento e do acompanhamento do trabalho de campo. F.A.F. CASTRO e R.C.G. ALFENAS participaram do planejamento, da orientação técnica em nutrição, do acompanhamento do trabalho e da revisão do manuscrito.

\section{REFERÊ NCIAS}

1. Barreto $S M$, Pinheiro $A R O$, Sichieri $R$, Monteiro CA, Batista Filho M, Schimidt Ml, et al. Análise da estratégia global para alimentação, atividade física e saúde, da Organização Mundial da Saúde. Epidemiol Serv Saúde. 2005; 14(1):41-68.

2. World Health Organization. Diet, nutrition and the prevention of chronic diseases. Report WHO 
Consultation. Geneva: WHO; 2003. WHO Technical Report Series, 916.

3. Malerbi DA, Franco LJ. Multicenter study of the prevalence of diabetes mellitus and impaired glucose tolerance in the urban Brazilian population aged 30-69 yr. Diabetes Care. 1992; 15(11): 1509-16.

4. Lotufo PA. Premature mortality from heart disease in Brazil: a comparison with other countries. Arq Bras Cardiol. 1998; 70(5):321-5. doi: 10.1590/ S0066-782X1998000500003.

5. Brasil. Ministério da Saúde. Diabetes responde por 70\% das amputações de assistência. [acesso 2005 dez 18]. Disponível em: <http://portalweb02. saude.gov.br/portal/aplicacoes/noticias/ noticias_detalhe.cfm?co_seq_noticia=22046>.

6. Brasil. Ministério da Saúde. Secretaria de Políticas Públicas. Plano de reorganização da atenção à hipertensão e ao diabetes mellitus: fase de detecção de casos suspeitos de DM. Rev Saúde Pública. 2001; 35(5):490-3. doi: 10.1590/\$003489102001000500014.

7. Georg AE, Ducan BB, Toscano CM, Schmidt MI, Mengue $S$, Duarte $C$, et al. Análise econômica de programa para rastreamento do diabetes mellitus no Brasil. Rev Saúde Pública. 2005; 39(3):452-60. doi: 10.1590/S0034-89102005000300017.

8. Pereira AMS. Análise dos resultados da campanha de detecção do diabetes ocorrida de março a abril de 2001, no Estado de Minas Gerais. Bol Epidemiol. 2001; 6(7):1-8.

9. Schaan BD, Harzheim E, Gus I. Perfil de risco cardíaco no Diabetes Melito e na glicemia de jejum alterada. Rev Saúde Pública. 2004; 38(4):529-36. doi: 10.1590/S0034-89102004000400008.

10. Zaitune MPA, Barros MBA, Cesar CLG, Carandina L, Goldbaum M. Hipertensão arterial em idosos: prevalência, fatores associados e práticas de controle no Município de Campinas. Cad Saúde Pública. 2006; 22(2):285-94. doi: 10.1590/S0102-31 1X2006000200006.

11. Simonetti JP, Batista L, Carvalho LR. Hábitos de salud y factores de riesgo en pacientes con hipertensión arterial. Rev Latino Am Enfermagem. 2002; 10(3): 415-22. doi: 10.1590/S0104-11692 002000300016.

12. Carvalho F, Telarolli Junior R, Machado JCS. Uma investigação antropológica na terceira idade: concepções sobre a hipertensão arterial. Cad Saúde Pública. 1998; 14(3):617-21. doi: 10.1590/S0102-31 $1 \times 1998000300019$.

13. Sociedade Brasileira de Hipertensão. V Diretrizes Brasileiras de Hipertensão Arterial. Tratamento não medicamentoso. São Paulo: SBH; 2006. p.48.
14. Dórea EL, Lotufo PA. Epidemiologia da hipertensão arterial sistêmica. Rev Hipertensão. 2004; 7(3): 86-9.

15. Luz PL, Cesena FHY. Prevenção da doença coronariana. Rev Soc Cardiol. 2001; 11(2):6-20.

16. Barreto SM, Passos VMA, Cardoso ARA, Lima-Costa MF. Quantifying the risk of coronary artery disease in a community: the Bambuí project. Arq Bras Cardiol. 2003; 81(6):556-61. doi: 10.1590/ S0066-782X2003001400002.

17. Organização Panamericana de Saúde. Doenças crônicas degenerativas e obesidade: estratégia mundial sobre alimentação, atividade física e saúde. Brasília: OPAS; 2003.

18. Stamber J, Stamber R, Neaton JD, Wentworth D, Daviglus ML, Garside D, et al. Low risk-factor profile and long-term cardiovascular and noncardiovascular mortality and life expectancy: findings for 5 large cahorts of young adult and midlle-ages men and women. J Am Med Assoc. 1999; 282(21): 2012-18.

19. Brasil. Ministério da Saúde. Secretaria de Políticas de Saúde. Plano de reorganização da atenção à hipertensão arterial e ao diabetes mellitus. Brasília; 2002.

20. Motta DG, Boog MCF. Fundamentos do comportamento alimentar. In: Educação nutricional. 3a. ed. São Paulo: Ibrasa. 1991. Capítulo 3, p.34-54.

21. Alves VS. Um modelo de educação em saúde para o programa saúde da família: pela integralidade da atenção e reorientação do modelo assistencial. Interface Comun Saúde Educ. 2005/2006; 9 (16-18):39-52. doi: 10.1590/\$1414-3283200500 0100004 .

22. Lima MT, Bucher JSNF, Lima JWO. A Hipertensão arterial sob o olhar de uma população carente: estudo exploratório a partir dos conhecimentos, atitudes e práticas. Cad Saúde Pública. 2004; 20(4):1079-87. doi: 10.1590/S0102-311X2004 000400023.

23. Fernandez PMF, Voci SM, Kamata LH, Najas MS, Souza ALM. Programa de saúde da família e as ações em nutrição em um distrito de saúde do município de São Paulo. Ciênc Saúde Coletiva. 2005; 10(3):749-55. doi: 10.1590/\$1413-812320 05000300031.

24. Assis AMO, Santos SMC, Freitas MCS, Santos JM, Silva MCM. O Programa saúde da família: contribuições para uma reflexão sobre a inserção do nutricionista na equipe multidisciplinar. Rev Nutr. 2002; 15(3):255-66. doi: 10.1590/S1415-52732 002000300001.

25. Brasil. Ministério da Saúde. Sistema de Informação de Atenção Básica. [acesso 2005 dez 12]. Disponível 
em: <http://www.datasus.gov.br/siab/siabs.htm? saude $=$ http $\% 3$ A \% 2F\%2Fwww. datasus.gov.br \%2Fsiab\%2Fsiabs.htm\&obj=\%24VObj\&botaook=OK>.

26. Hulley SB, Gove S, Browner WS, Cummings SR. Elección de los indivíduos que participarán en el estudio: especificación y muestreo. In: Hulley SB, Cummings SR, Browner WS, Newman TB, Hearst $N$. Diseño de la investigación clínica: um enfoque epidemiológico. Madrid: Harcourt Brace; 1997.

27. Richardson RJ. Pesquisa social: métodos e técnicas. 3a. ed. São Paulo: Atlas; 1999.

28. Philippi ST, Latterza AR, Cruz ATR, Ribeiro LC. Pirâmide alimentar adaptada: guia para escolha dos alimentos. Rev Nutr. 1999; 12(1):65-80. doi: 10.1590/S1415-52731999000100006.

29. Brasil. Ministério da Saúde. Guia alimentar: saiba como ter uma alimentação saudável. [acesso 2008 jul 20]. Disponível em: <http://portal.saude.sp. gov.br/resources/cidadao/destaques/guia_de_ bolso_sobre_alimentacao.pdf>.

30. Pallas JMA, Villa JJ. Métodos de investigacion: aplicados a la atención primaria de salud. 2a. ed. Madrid: Mosby/ Doyma; 1995.

31. Oliveira MC, Sichieri R. Fracionamento das refeições e colesterol sérico em mulheres com dieta adicionada de frutas ou fibras. Rev Nutr. 2004; 17(4):449-59. doi: 10.1590/S1415-52732004 000400005.

32. Costa EQ, Alves AR, Pires CC. Avaliação do comportamento de idosos perante os alimentos dietéticos. Anais do $7^{\circ}$ Congresso da Sociedade Brasileira de Alimentação e Nutrição: Alimentação e Nutrição: avanços tecnológicos e desafios éticos; 2003 out 15-18; Belo Horizonte. p.68.

33. Franz MJ, Bantle JP, Beebe CA, Brunzell JD, Chiasson JL, Garg A, et al. American Diabetes Association. Nutrition principles and recommendations in diabetes. Diabetes Care. 2004; 27(Suppl 1):S36-46.

34. Atkinson FS, Foster-Powell K, Brand-Miller JC. International Tables of Glycemic Index and Glycemic Load Values: 2008. Diabetes Care. 2008; 31(12):2281-83. doi: $10.2337 / \mathrm{dc0} 8-1239$.

35. Seara LT, Amorim NCS, Albuquerque MFM. Uso de adoçantes artificiais de adição e de produtos dietéticos e freqüentadores/as de clubes de melhor idade de Maceió - AL. Anais do $7^{\circ}$ Congresso da Sociedade Brasileira de Alimentação e Nutrição: Alimentação e Nutrição: avanços tecnológicos e desafios éticos; 2003 out. 15-18; Belo Horizonte. p.70.

36. Castro AGP, Franco LJ. Caracterização do consumo de adoçantes alternativos e produtos dietéticos por indivíduos diabéticos. Arq Bras Endocrinol Metab. 2002; 46(3):280-7. doi: 10.1590/S000427302002000300011.

37. Bezerra TM, Pires ISC, Esteves EL, Falqueto ICC, Amorim YJ, Scaramussa L, et al. Avaliação sensorial de adoçantes em preparações de doces. Anais do $7^{\circ}$ Congresso da Sociedade Brasileira de Alimentação e Nutrição: Alimentação e Nutrição: avanços tecnológicos e desafios éticos. 2003 out. 15-18; Belo Horizonte. p.43.

38. Piccini RX, Victoria CG. Hipertensão arterial sistêmica em área urbana no sul do Brasil: prevalência e fatores de risco. Rev Saúde Pública. 1994; 28(4):261-7. doi: 10.1590/S0034-89101994000 400004.

39. Tavares A, Kohemanm Jr O. Tratamento da hipertensão arterial: valor da redução na ingestão de sal. Rev Hipertensão. 2004; 7(2):71-3.

40. Levy-Costa RB, Sichieri R, Pontes NS, Monteiro CA. Disponibilidade domiciliar de alimentos no Brasil: distribuição e evolução (1974-2003). Rev Saúde Pública. 2005; 39(4):530-40. doi: 10.1590/\$003489102005000400003.

41. Sartorelli DS, Franco LJ. Tendências do diabetes mellitus no Brasil: o papel da transição nutricional. Cad Saúde Pública. 2003; 19(Sup.1):S29-36. doi: 10.1590/S0102-311X2003000700004.

42. Pereira LO, Francischi RP, Lancha Jr AH. Obesidade: hábitos nutricionais, sedentarismo e resistência à insulina. Arq Bras Endocrinol Metabol. 2003; 47(2):111-27. doi: 10.1590/50004-27302003000 200003.

43. Guimarães FPM, Takayanagui AMM. Orientações recebidas do serviço de saúde por pacientes para o tratamento do portador de diabetes mellitus tipo 2. Rev Nutr. 2002; 15(1):37-44. doi: 10.1590/S14 15-52732002000100005.

44. Brasil. Instituto Brasileiro de Geografia e Estatística. Perfil dos Municípios Brasileiros. Cultura 2006. [acesso 2009 mar 7]. Disponível em: <http:// www.ibge.gov.br/home/presidencia/noticias/ noticia_visualiza.php?id_noticia=980>.

Recebido em: 11/12/2007

Versão final reapresentada em: 30/4/2009

Aprovado em: 7/7/2009 